\title{
Comprehensive cardiac magnetic resonance imaging and spectroscopy reveals a high burden of myocardial disease in HIV infection
}

\author{
Cameron Holloway*, Ntobeko Ntusi, Joseph Suttie, Masliza Mahmod, Emma Wainwright, Genevieve Clutton, \\ Gemma Hancock, Philip Beak, Abdelouahid Tajar, Stefan K Piechnik, Jurgen E Schneider, Kieran Clarke, \\ Lucy Dorrell, Stefan Neubauer
}

From 16th Annual SCMR Scientific Sessions San Francisco, CA, USA. 31 January - 3 February 2013

\section{Background}

Human immunodeficiency virus (HIV) infection continues to be endemic worldwide. Whilst treatments are successful, it remains controversial whether patients receiving optimal therapy for HIV infection have structural, functional or biochemical cardiac abnormalities which may underlie the increased cardiac morbidity and mortality. Our main objective was to characterise myocardial abnormalities in a contemporary group of HIV-infected individuals.

\section{Methods}

This was a cross-sectional observational study of patients with HIV infection. One hundred and forty three volunteers were recruited in this study, among those 104 were asymptomatic HIV-infected subjects aged $\geq 18$ years (91 receiving cART) without a history of cardiovascular disease. They underwent cardiac magnetic resonance imaging (MRI) and spectroscopy (MRS). Cardiovascular risk factors, duration of HIV infection and cART and fasting plasma metabolite levels were recorded for each subject. Myocardial fibrosis, cardiac function and myocardial lipid content were assessed by MRI and MRS.

\section{Results}

Compared to age-matched controls, HIV-infected subjects had on average $54 \%$ higher myocardial lipids, together with a two-fold elevation in plasma triglycerides (both $\mathrm{p}<$ 0.01 ). Myocardial fibrosis, predominantly in the basal infero-lateral wall of the left ventricle, was observed in $78 \%$ of HIV-infected subjects compared to $13 \%$ of controls
( $\mathrm{p}<0.001$ ), irrespective of duration of infection or cART exposure. Peak myocardial systolic and diastolic longitudinal strain were also lower in HIV-infected individuals compared to controls (9\% and $27 \%$ respectively, both $\mathrm{p}<$ $0.01)$, and so was left ventricular ejection fraction $(68 \pm 1 \%$ vs. $72 \pm 1 \%, \mathrm{p}<0.05)$. The differences in myocardial steatosis and fibrosis remained even after adjusting for potential confounders.

\section{Conclusions}

Comprehensive Cardiac Magnetic Resonance imaging and spectroscopy revealed cardiac steatosis, alterations in cardiac function and a high prevalence of myocardial fibrosis in a contemporary group of asymptomatic HIVinfected subjects. Cardiac steatosis and fibrosis may underlie cardiac dysfunction and increased cardiovascular morbidity and mortality in subjects living with HIV.

\section{Funding}

Oxford NIHR Biomedical Research Centre programme. BHF Centre of Research. The British Heart Foundation (FS07/030) supported this work.

Published: 30 January 2013

doi:10.1186/1532-429X-15-S1-O25

Cite this article as: Holloway et al: Comprehensive cardiac magnetic resonance imaging and spectroscopy reveals a high burden of myocardial disease in HIV infection. Journal of Cardiovascular Magnetic Resonance 2013 15(Suppl 1):O25.

University of Oxford, Oxford, UK

๑ 2013 Holloway et al; licensee BioMed Central Ltd. This is an Open Access article distributed under the terms of the Creative 